\title{
High-Accuracy Analysis of Compton Scattering in Chiral Effective Field Theory: Status and Future
}

\author{
Harald W. Grießhammer*, Daniel R. Phillips ${ }^{\dagger}$ and Judith A. McGovern** \\ *Institute for Nuclear Studies, Department of Physics, George Washington University, Washington DC 20052, USA \\ and IKP-3, IAS and Jülich Centre for Hadron Physics, Forschungszentrum Jülich, D-52428 Jülich, Germany \\ ${ }^{\dagger}$ Dept. of Physics and Astronomy, Inst. of Nuclear and Particle Physics, Ohio University, Athens OH 45701, USA \\ ${ }^{*}$ School of Physics and Astronomy, The University of Manchester, Manchester M13 9PL, UK
}

\begin{abstract}
Theory and prospects of Compton scattering on nucleons and light nuclei below $500 \mathrm{MeV}$ are outlined; cf. [1-3]. Invited contribution at the WORKSHOP TO EXPLORE PHYSICS OPPORTUNITIES WITH INTENSE, POLARIZED ELECTRON BeAmS With ENERGY UP TO 300 MeV, MIT, Cambridge (USA), 14th-16th March 2013.
\end{abstract}

Keywords: Compton scattering, nucleon polarisabilities, spin polarisabilities, polarised experiments, Chiral Effective Field Theory, $\Delta(1232)$

\section{WHY COMPTON SCATTERING?}

In Compton scattering $\gamma \mathrm{X} \rightarrow \gamma \mathrm{X}$, the electric and magnetic fields of a real photon induce radiation multipoles by displacing the charged constituents and currents inside the target. The energy- and angle-dependence of the emitted radiation explores the interactions of its constituents. In Hadronic Physics, it elucidates the distribution, symmetries and dynamics of the charges and currents which constitute the low-energy degrees of freedom inside the nucleon, and - for few-nucleon systems - the interactions between nucleons; see a recent review for details [1]. The 2007 NSAC and 2010 NuPECC Long-Range Plans emphasise therefore the pivotal rôle of the nucleon's temporal two-photon response below $1 \mathrm{GeV}$ to complement the information accessible in one-photon experiments like form-factor measurements. As a consequence, a number of experiments are presently being pursued at MAMI (Mainz) [4], HI $\gamma \mathrm{S}$ at TUNL [5], and MAX-Lab at Lund [6]. For the next generation, Aron Bernstein and Rory Miskimen have shown at this workshop how to use high-intensity electron beams like MESA for "near-real" photon experiments [4, 7].

In contradistinction to many electro-magnetic processes, such structure effects have only recently been subjected to a multipole-analysis. The Fourier transforms of the corresponding temporal response functions are the proportionality constants between incident field and induced multipole. These energy-dependent polarisabilities parametrise the stiffness of the nucleon $N$ (spin $\frac{\vec{\sigma}}{2}$ ) against transitions $X l \rightarrow Y l^{\prime}$ of given photon multipolarity at fixed frequency $\omega\left(l^{\prime}=l \pm\{0 ; 1\} ; X, Y=E, M ; T_{i j}=\frac{1}{2}\left(\partial_{i} T_{j}+\partial_{j} T_{i}\right) ; T=E, B\right)$. Up to $500 \mathrm{MeV}$, the relevant terms are:

$$
\begin{aligned}
\mathscr{L}_{\mathrm{pol}}=2 \pi N^{\dagger}[ & \alpha_{E 1}(\omega) \vec{E}^{2}+\beta_{M 1}(\omega) \vec{B}^{2}+\gamma_{E 1 E 1}(\omega) \vec{\sigma} \cdot(\vec{E} \times \dot{\vec{E}})+\gamma_{M 1 M 1}(\omega) \vec{\sigma} \cdot(\vec{B} \times \dot{\vec{B}}) \\
& \left.\left.-2 \gamma_{M 1 E 2}(\omega) \sigma^{i} B^{j} E_{i j}+2 \gamma_{E 1 M 2}(\omega) \sigma^{i} E^{j} B_{i j}+\ldots \text { (photon multipoles beyond dipole }\right)\right] N
\end{aligned}
$$

The two spin-independent polarisabilities $\alpha_{E 1}(\omega)$ and $\beta_{M 1}(\omega)$ parametrise electric and magnetic dipole transitions. Of particular interest are at present the four dipole spin-polarisabilities $\gamma_{E 1 E 1}(\omega), \gamma_{M 1 M 1}(\omega), \gamma_{E 1 M 2}(\omega), \gamma_{M 1 E 2}(\omega)$. They encode the response of the nucleon spin-structure, i.e. of the spin constituents. Intuitively interpreted, the electromagnetic field associated with the spin degrees causes bi-refringence in the nucleon, just like in the classical Faraday-effect. Only the linear combinations $\gamma_{0}$ and $\gamma_{\pi}$ of scattering under $0^{\circ}$ and $180^{\circ}$ are somewhat constrained by data or phenomenology, with conflicting results for the proton (MAMI, LEGS) and large error-bars for the neutron.

The values $\alpha_{E 1}(\omega=0)$ etc. are often called "the (static) polarisabilities"; but the $\omega$-dependence reveals more. Since the polarisabilities are the parameters of a multipole decomposition, they contain not more information than the full amplitudes, but characteristic signatures in specific channels are easier to interpret. For example, the significant $\omega$-dependence of $\beta_{M 1}(\omega)$ and $\gamma_{M 1 M 1}(\omega)$ already for $\omega \gtrsim 100 \mathrm{MeV}$ comes from the strong para-magnetic $\gamma \mathrm{N} \Delta(1232)$ transition. The $\Delta(1232)$ enters thus dynamically well below the resonance region. The electric polarisabilities exhibit a pronounced cusp at the pion-production threshold. As soon as an inelastic channel opens, polarisabilities become complex. Thus, their imaginary parts provide an alternative to explore pion-photoproduction multipoles. Polarisabilities 
also enter as one of the bigger sources of uncertainties in theoretical determinations of the proton-neutron mass difference (see e.g. most recently [8]), and of the two-photon-exchange contribution to the Lamb shift in muonic hydrogen (see e.g. most recently [9]). Finally, nuclear targets provide an opportunity to study not only neutron polarisabilities, but also the nuclear force directly, since the photons couple to the charged pion-exchange currents in the nucleus.

\section{CHIRAL EFFECTIVE FIELD THEORY $\chi$ EFT IN COMPTON SCATTERING}

Interpreting such data of course requires commensurate theoretical support. One must carefully evaluate dataconsistency in one model-independent framework for hidden systematic errors; subtract binding effects in few-nucleon systems; extract the polarisabilities using minimal theoretical bias; identify the underlying QCD mechanisms, like the detailed chiral dynamics of the pion cloud and of the $\Delta(1232)$ resonance; relate them to emerging lattice QCD simulations - and do all of that while providing reproducible theoretical uncertainties.

$\chi$ EFT, the low-energy theory of QCD and extension of Chiral Perturbation Theory to few-nucleon systems, has been quite successful in proton and few-nucleon Compton scattering, starting with the parameter-free leading-order prediction $\alpha_{E 1}=10 \beta_{M 1}=12.4 \times 10^{-4} \mathrm{fm}^{3}$ by Bernard et al. [10]. $\chi$ EFT generates the most general Compton amplitude consistent with gauge invariance, the pattern of chiral-symmetry breaking in QCD, and Lorentz covariance. A particularly interesting $\chi$ EFT prediction is that small proton-neutron differences in polarisabilities stem from chiralsymmetry breaking $\pi \mathrm{N}$ interactions and thus probe details of QCD. In $\chi$ EFT with explicit $\Delta(1232)$ degrees of freedom, the low-energy scales are the pion mass $m_{\pi} \approx 140 \mathrm{MeV}$ as the typical chiral scale; the Delta-nucleon mass splitting $\Delta_{M} \approx 290 \mathrm{MeV}$; and the photon energy $\omega$. When measured in units of a natural "high" scale $\Lambda \gg \Delta_{M}, m_{\pi}, \omega \approx$ $800 \mathrm{MeV}$ at which this variant can be expected to break down because new degrees of freedom become dynamical, each gives rise to a small, dimensionless expansion parameter. In the $\delta$-expansion of Pascalutsa and Phillips [11], one avoids a threefold expansion by approximately relating scales so that only one dimensionless parameter is left:

$$
\delta \equiv \frac{\Delta_{M}}{\Lambda} \approx\left(\frac{m_{\pi}}{\Lambda}\right)^{1 / 2}
$$

i.e. numerically $\delta \approx 0.4$. For $\omega \sim m_{\pi}$, the Thomson amplitude is leading-order, $\mathscr{O}\left(e^{2} \delta^{0}\right)$; structure effects start with $\pi \mathrm{N}$ loops at $\mathscr{O}\left(e^{2} \delta^{2}\right)$; and since $\Delta_{M} \sim \delta$ whereas $m_{\pi} \sim \delta^{2}, \pi \Delta$ loops are suppressed by an additional power to $\mathscr{O}\left(e^{2} \delta^{3}\right)$.

The Delta-pole graph is $\mathscr{O}\left(e^{2} \delta^{3}\right)$ for $\omega \sim m_{\pi}$, but its enhancement close to the Delta's on-shell point leads to a re-ordering of contributions at higher energies, $\omega \sim \Delta_{M}$. The $\pi \mathrm{N}$ loops that generate the resonance's nonzero width must then be resummed. In this régime, the dominant Compton mechanism, $\mathscr{O}\left(e^{2} \delta^{-1}\right)$, is the excitation of a dressed $\Delta(1232)$ by the magnetic transition from the nucleon, followed by de-excitation via the same M1 transition. The E2 $\mathrm{N} \rightarrow \Delta(1232)$ transition and leading-one-loop corrections to the $\gamma \mathrm{N} \Delta$ vertex enter at $\mathscr{O}\left(e^{2} \delta^{0}\right)$. Relativistic kinematics is of course essential around the $\Delta$ resonance. Recently, single-nucleon Compton amplitudes were derived which apply from zero energy to about $400 \mathrm{MeV}$. For $\omega \lesssim m_{\pi}$, they contain all contributions at $\mathscr{O}\left(e^{2} \delta^{4}\right)\left(\mathrm{N}^{4} \mathrm{LO}\right.$, accuracy $\delta^{5} \lesssim 2 \%$ ), and for $\omega \sim \Delta_{M}$ all at $\mathscr{O}\left(e^{2} \delta^{0}\right)$ (NLO, accuracy $\delta^{2} \lesssim 20 \%$ ); see Refs. [1, 2] for a detailed discussion. Vladimir Pascalutsa's talk presented results of an alternative, manifestly covariant $\chi$ EFT variant [12].

For light nuclei, $\chi$ EFT provides at present deuteron Compton scattering results which are complete at $\mathscr{O}\left(e^{2} \delta^{3}\right)$ from the Thomson limit up to about $120 \mathrm{MeV}$, including the $\Delta(1232)$ degree of freedom $[1,13]$. This is now being extended both to higher energies and by including the new single-nucleon amplitudes. For ${ }^{3} \mathrm{He}$, the results in a variant without explicit $\Delta(1232)$ and for $\omega \in[80 ; 120] \mathrm{MeV}$ [14] are extended, too. In few-nucleon systems, the Thomson limit as exact low-energy theorem is a result of NN rescattering between photon emission and absorption. While computationally intensive, its rôle diminishes for $\omega \gtrsim 100 \mathrm{MeV}$ because the photon does not scatter any more coherently from the target nucleus as a whole. Finally, nuclei themselves are made of charged particles which are displaced by the photon fields and thus have an intrinsic polarisability. For the deuteron, these are known on the $\lesssim 1 \%$-level, with various EFT variants and conventional calculations agreeing very well [1].

\section{NEW STATIC POLARISABILITIES FROM $\chi$ EFT}

In the unified single-nucleon amplitudes described above, the $\pi \mathrm{N}$ parameters take their standard values [1, 2]. The $\Delta(1232)$ parameters $\Delta_{M}=293 \mathrm{MeV}$ and $g_{\pi \mathrm{N} \Delta}=1.425$ are obtained from the Breit-Wigner peak and width via the relativistic formula, and the ratio of E2 and M1 couplings is $b_{2} / b_{1}=-0.34$. Two contact interactions encode the 
short-distance $\left(r \ll 1 / m_{\pi}, 1 / \Delta_{M}\right)$ contributions to the scalar polarisabilities. Their coefficients (or, equivalently, the static values $\alpha_{E 1}$ and $\beta_{M 1}$ ) must be fitted. To that end, one must first establish a statistically consistent database from all available proton data below $350 \mathrm{MeV}$ in Ref. [1], carefully pruning the data by objective and transparent criteria.

Since the power counting confirms that the high-energy amplitudes are most sensitive to $\Delta$ parameters, the $\gamma \mathrm{N} \Delta \mathrm{M} 1$ coupling $b_{1}$ is determined from the MAMI data for $\omega_{\mathrm{lab}}=200-325 \mathrm{MeV}$. Sensitivity to the polarisabilities is greater at $\omega \lesssim 170 \mathrm{MeV}$, where the amplitudes are also known with higher accuracy. Thus, $\alpha_{E 1}^{(\mathrm{p})}$ and $\beta_{M 1}^{(\mathrm{p})}$ are fit concurrently to these low-energy data, with iteration betwixt both regions until convergence is reached. One finds a solution with a $\chi^{2} /$ d.o.f. $=113.2 / 135, b_{1}=3.61 \pm 0.02$ and the static scalar proton polarisabilities as (stat. errors from $\left.\chi^{2}+1\right)$ [2]:

$$
\alpha_{E 1}^{(\mathrm{p})}=\left[10.7 \pm 0.4_{\text {stat }} \pm 0.2_{\text {Baldin }} \pm 0.3_{\text {theory }}\right] \times 10^{-4} \mathrm{fm}^{3}, \beta_{M 1}^{(\mathrm{p})}=\left[3.1 \mp 0.4_{\text {stat }} \pm 0.2_{\text {Baldin }} \mp 0.3_{\text {theory }}\right] \times 10^{-4} \mathrm{fm}^{3}
$$

Since a fit to $\alpha_{E 1}$ and $\beta_{M 1}$ independently is highly consistent with the Baldin sum rule $\alpha_{E 1}^{(\mathrm{p})}+\beta_{M 1}^{(\mathrm{p})}=[13.8 \pm 0.4] \times$ $10^{-4} \mathrm{fm}^{3}$, the numbers quoted above use this constraint. All fits are stable under reasonable variations in the procedure and agree with the data well beyond the region in which the parameters are determined. Special care has been taken to reproducibly justify a theoretical uncertainty of $\pm 0.3 \times 10^{-4} \mathrm{fm}^{3}$ from the most conservative of several estimates of higher-order terms. For an acceptable fit, $\gamma_{M 1 M 1}$ is treated as parameter, albeit its counter term enters at higher order.

Neutron polarisabilities are extracted from the elastic deuteron data base of Ref. [1]. It has significantly larger statistical error-bars and is only a tenth of the size of the proton one, with data at only a few angles and energies $\omega \in[49 ; 94] \mathrm{MeV}$. These amplitudes are one order lower than in the proton extraction, but the statistical errors are still larger than the estimated theoretical uncertainties. The correct Thomson limit and NN rescattering is important also to reduce residual dependence on the choice of the deuteron wave function to $<1 \%$ [1]. The fit to the isoscalar, scalar dipole polarisabilities yields with $\chi^{2} /$ d.o.f. $=24.3 / 25$ :

$$
\begin{aligned}
& \alpha_{E 1}^{(\mathrm{s})}=\left[10.9 \pm 0.9_{\text {stat }} \pm 0.2_{\text {Baldin }} \pm 0.8_{\text {theory }}\right] \times 10^{-4} \mathrm{fm}^{3}, \beta_{M 1}^{(\mathrm{s})}=\left[3.6 \mp 0.9_{\text {stat }} \pm 0.2_{\text {Baldin }} \mp 0.8_{\text {theory }}\right] \times 10^{-4} \mathrm{fm}^{3} \\
& \alpha_{E 1}^{(\mathrm{n})}=\left[11.1 \pm 1.8_{\text {stat }} \pm 0.2_{\text {Baldin }} \pm 0.8_{\text {theory }}\right] \times 10^{-4} \mathrm{fm}^{3}, \beta_{M 1}^{(\mathrm{n})}=\left[4.2 \mp 1.8_{\text {stat }} \pm 0.2_{\text {Baldin }} \mp 0.8_{\text {theory }}\right] \times 10^{-4} \mathrm{fm}^{3}
\end{aligned}
$$

The $\chi^{2}+1$ ellipsoid is again the statistical error. In the last line, the proton and isoscalar values are combined to the static scalar neutron polarisabilities. An independent fit to $\alpha_{E 1}^{(\mathrm{s})}$ and $\beta_{M 1}^{(\mathrm{s})}$ is again consistent with the (isoscalar) Baldin sum rule, so this constraint reduces statistical uncertainties. In contrast to the proton case, the data are consistent: each experiment contributes roughly equally to the $\chi^{2}$, and the extracted polarisabilities are largely insensitive to the elimination of any one data set. Within the statistics-dominated errors, the proton and neutron polarisabilities are thus identical, i.e. no isospin breaking effects of the pion cloud are seen, as predicted by Chiral EFT. In all cases, the normalisation of each data set is floated within the quoted normalisation uncertainty; Refs. [1,2] give more details.
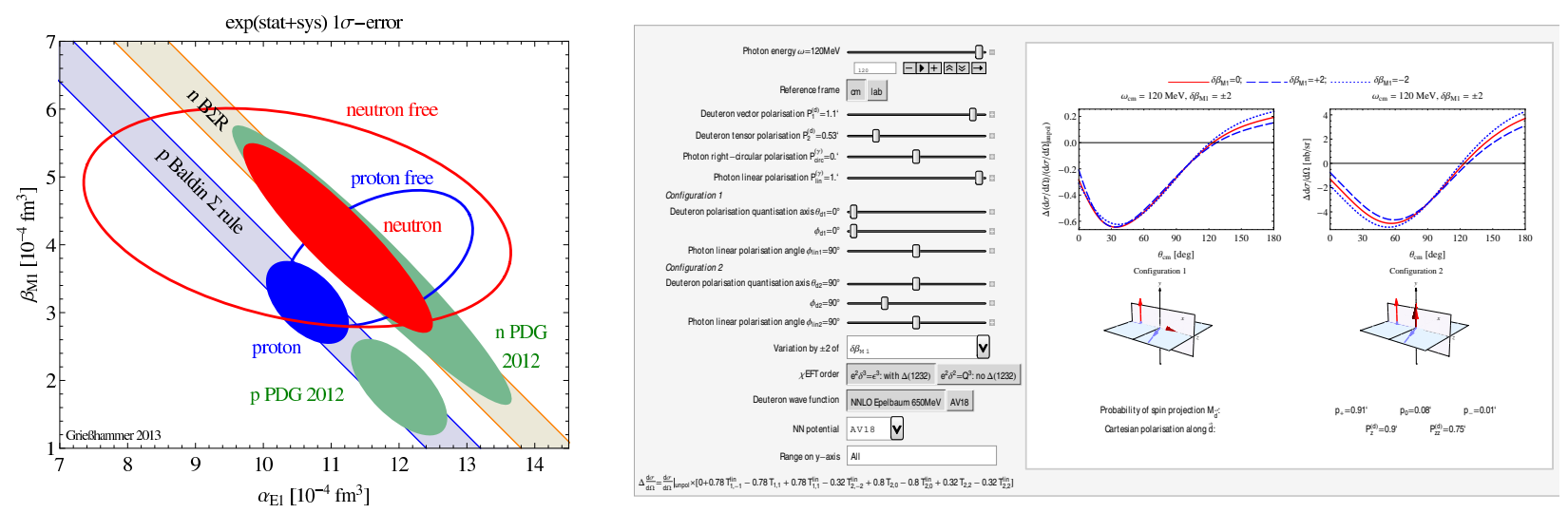

FIGURE 1. Left: Comparison of static scalar dipole polarisability values in $\chi$ EFT from Refs. [1, 2] and PDG values (green). Blue: proton; red: neutron; disks (ellipses): with (without) Baldin Sum Rule constraints. Notice that errors are statistical only, and $1 \sigma$, not $\chi^{2}+1$. Right: Screen-shot of a Mathematica notebook for $\gamma \mathrm{d}$ scattering with arbitrary polarisations; from Ref. [3]. 


\section{OPPORTUNITIES FOR HIGH-ACCURACY, HIGH-LUMINOSITY EXPERIMENTS}

The future lies in un-, single- and double-polarised experiments of high accuracy, with reproducible systematic uncertainties; see [4-6] for ongoing and planned efforts. For unpolarised experiments on the proton, the greatest impact is where the data base of nearly 300 points is most scarce: (1) at forward angles (test the Baldin sum rule); (2) at extreme back-angles (test $\alpha_{E 1}-\beta_{M 1}$ ); (3) between 170 and $240 \mathrm{MeV}$ (sparse and inconsistent data); (4) to resolve the several- $\sigma$ discrepancy between LEGS and MAMI which prevents a consistent fit to all sets simultaneously [2].

In order to understand the subtle differences of the pion clouds around the proton and neutron induced by explicit chiral symmetry breaking in QCD, we need the neutron polarisabilities with uncertainties comparable to those of the proton. MAXlab and HI $\gamma \mathrm{S}$ are aiming to augment the angular and energy range of the 29 unpolarised data points for the deuteron with statistical and systematic uncertainties of better than $5 \%$. Both also have plans for ${ }^{3} \mathrm{He}$. First results on unpolarised ${ }^{6} \mathrm{Li}$ have been reported, and theory is starting to catch up [15]. Such targets are experimentally better to handle and provide count rates which scale at least linearly with the target charge when photons scatter incoherently from the protons, i.e. $\omega \gtrsim 100 \mathrm{MeV}$. However, describing the energy levels of these nuclei with adequate accuracy is theoretically involved. For the proton, amplitudes on the $\lesssim 2 \%$-level are available; for deuteron and ${ }^{3} \mathrm{He}$, consistent Compton amplitudes from zero energy into the Delta resonance region are being developed in $\chi$ EFT. Around ${ }^{3} \mathrm{He}-$ ${ }^{4} \mathrm{He}-{ }^{6} \mathrm{Li}$ may well be the "sweet-spot" between needs and wants of theorists and experimentalists. Neutron values can also be isolated both in quasi-free kinematics and from polarised ${ }^{3} \mathrm{He}$, which effectively is a free-neutron target [14].

The highest impact of high-intensity (near-real) photon beam machines will however be in the study of the so-far nearly untested spin-polarisabilities: four each for the proton and neutron. Since they test the spin-constituents of the nucleon, they are a top priority of experiment and theory alike. Sensitivity studies have been performed in $\chi$ EFT variants with and without explicit $\Delta(1232)$; see summary in [1, Sec. 6.1] and V. Pascalutsa's talk for a covariant $\chi$ EFT variant [12]. Asymmetries remove many systematic effects. Recently, the cross section with arbitrary photon and deuteron polarisation has for example been parametrised via 18 independent observables [3]. An exploration of the sensitivity of each on the nucleon's scalar and spin dipole polarisabilities in the $\chi$ EFT variant discussed above shows that some asymmetries are sensitive to only one or two dipole polarisabilities. This makes them particularly attractive for an energy-dependent multipole-expansion of Compton scattering; cf. [1]. For spin polarisabilities with an error of $\pm 2 \times 10^{-4} \mathrm{fm}^{4}$, asymmetries should be measured with an accuracy of $\gtrsim 10^{-2}$, with differential cross sections of a dozen $\mathrm{nb} / \mathrm{sr}$ at $100 \mathrm{MeV}$ or a few dozen $\mathrm{nb} / \mathrm{sr}$ at $250 \mathrm{MeV}$. Relative to single-nucleon Compton scattering, interference with the $D$ wave and the pion-exchange current of the deuteron increases the sensitivity to the "mixed" spin polarisabilities $\gamma_{E 1 M 2}$ and $\gamma_{M 1 E 2}$. One may thus speculate that their determination will first appear from deuteron data - and high-intensity beams can provide the necessary accuracy. A Mathematica 9.0 file for $\omega<120 \mathrm{MeV}$ is available from hgrie@gwu. edu; see screen-shot in Fig. 1. Parallel studies for the proton and ${ }^{3} \mathrm{He}$ are under way.

\section{ACKNOWLEDGMENTS}

HWG cordially thanks the organisers for a stimulating atmosphere and financial support. Work supported in part by UK Science and Technology Facilities Council grants ST/F012047/1, ST/J000159/1 (JMcG) and ST/F006861/1 (DRP), by US Department of Energy grants DE-FG02-95ER-40907 (HWG) and DE-FG02-93ER-40756 (DRP), by the Deutsche Forschungsgemeinschaft and the National Natural Science Foundation of China via the Sino-German CRC 110 "Symmetries and the Emergence of Structure in QCD" (HWG), and by the EPOS network of the European Community Research Infrastructure Integrating Activity "Study of Strongly Interacting Matter" (HadronPhysics3).

\section{REFERENCES}

1. H. W. Grießhammer, J. A. McGovern, D. R. Phillips and G. Feldman, Prog. Part. Nucl. Phys. 67, 841 (2012).

2. J. A. McGovern, D. R. Phillips, and H. W. Grießhammer, Eur. Phys. J. A49 (2013) 12

3. H. W. Grießhammer, arXiv:1304.6594 [nucl-th].

4. R. Miskimen, these proceedings.

5. H. R. Weller et al., AIP Conf. Proc. 1182, 890 (2009); exp. E-06-09, E-06-10 (p), E-18-09 (d), E-07-10 ( $\left.{ }^{3} \mathrm{He}\right)$, E-15-11 ( $\left.{ }^{6} \mathrm{Li}\right)$.

6. G. Feldman et al., Few Body Syst. 44, 325 (2008); $\gamma d$ at 100-200 MeV.

7. A. Bernstein, these proceedings.

8. A. Walker-Loud, C. E. Carlson and G. A. Miller, Phys. Rev. Lett. 108 (2012) 232301.

9. M. C. Birse and J. A. McGovern, Eur. Phys. J. A 48 (2012) 120. 
10. V. Bernard, N. Kaiser and U. G. Meißner, Phys. Rev. Lett. 67 , 1515 (1991); Int. J. Mod. Phys. E 4 (1995) 193.

11. V. Pascalutsa and D. R. Phillips, Phys. Rev. C 67, 055202 (2003).

12. V. Pascalutsa, these proceedings.

13. R. P. Hildebrandt, H. W. Grießhammer and T. R. Hemmert, Eur. Phys. J. A 46, 111 (2010).

14. D. Shukla, A. Nogga and D. R. Phillips, Nucl. Phys. A 819 (2009) 98.

15. G. Bampa, W. Leidemann and H. Arenhovel, Phys. Rev. C 84 (2011) 034005; W. Leidemann, priv. comm. 